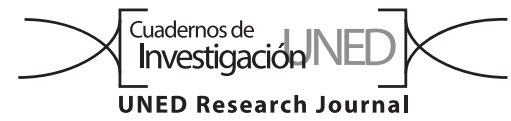

\title{
Satisfacción con la vida, el trabajo, y el país, en funcionarios de una universidad costarricense: comparación de sede central y centros regionales
}

\author{
Lourdes Arce Espinoza' \& Karla Rojas Sáurez ${ }^{1}$ \\ 1. Universidad Estatal a Distancia, Vicerrectoría Ejecutiva, Servicio Médico UNED, 2050 Sabanilla, San José, Costa Rica; \\ larce@uned.ac.cr, (iD https://orcid.org/0000-0003-0681-0867; krojas@uned.ac.cr, (DD https://orcid.org/0000-0002-9110-3835 \\ Recibido 11-VII-2019 • Corregido 20-VIII-2019 • Aceptado 27-X-2019 \\ DOI: https://doi.org/10.22458/urj.v11i3.2721
}

\begin{abstract}
Satisfaction with life, work, and country, in staff of a Costa Rican university: comparison of headquarters and regional centers". Introduction: Satisfaction has been the subject of study by various disciplines, whose interest is to determine which factors or variables make their grade higher in an individual or in another if they have the same environmental and environmental conditions. The level of satisfaction is subjective and will depend to a great extent on the attitudes of the individual in relation to the extrinsic factors that surround him. Objective: To compare the level of satisfaction in the officials of the central headquarters and the university centers of a state university of Costa Rica. Methods: In the present study, we measured satisfaction in three dimensions: aspects of life, work and national scope, from which an index of personal satisfaction is calculated; the results obtained are compared between the University Center officials and the officials of the Headquarters of a state university of Costa Rica. Results: The level of satisfaction is higher in the capital, except for work, where there is no difference. Conclusions: The economic disadvantage in the regional centers matches a less optimistic view of the country and a lower level of personal satisfaction with life.
\end{abstract}

Key words: Well-being, lifestyle, quality of life, happiness, mental health, achievements.
RESUMEN: Introducción: La satisfacción ha sido objeto de estudio por diversas disciplinas, cuyo interés es poder determinar qué factores o variables hacen que su grado sea más alto en un individuo o en otro si tienen las mismas condiciones de ambiente y entorno. El nivel de satisfacción es subjetivo y dependerá en gran medida de las actitudes del individuo en relación con los factores extrínsecos que lo rodean. Objetivo: Comparar el nivel de satisfacción en los funcionarios de la Sede Central y los Centros universitarios de una universidad estatal de Costa Rica. Métodos: En el presente estudio medimos la satisfacción en tres dimensiones: aspectos de la vida, laboral y ámbito nacional, a partir de los cuales se calcula un índice de satisfacción personal; los resultados obtenidos se comparan entre los funcionarios de Centros Universitarios y los funcionarios de la Sede Central de una universidad estatal de Costa Rica. Resultados: El nivel de satisfacción es mayor en la capital, excepto por trabajo, donde no hay diferencia. Conclusiones: La desventaja económica en los centros regionales coincide con una visión menos optimista del país y un menor nivel de satisfacción personal con la vida.

Palabras clave: Bienestar, estilo de vida, calidad de vida, felicidad, salud mental, logros.
La satisfacción ha sido de interés en muchas disciplinas o ramas de la ciencia tales como: la psicología, sociología, administración, economía y finanzas, entre otras. En el año 1954 se introduce la Teoría de la Pirámide de Maslow y en 1959 la Teoría de la Motivación e Higiene, a partir de estas teorías se derivan otras que tratan de explicar el mecanismo de la satisfacción, todas coinciden en que existen factores intrínsecos y extrínsecos que determinan el nivel de satisfacción de los individuos (Téllez, 2011; Benedicto, 2013; Duarte, Aguirre, \& Jarquín, 2014).

El balance entre lo esperado y lo obtenido, es lo que muchos autores consideran determina el grado de satisfacción de un individuo. Esto sumado también a los factores intrínsecos y extrínsecos que forman la totalidad del ambiente del individuo, da como resultado mayor o menor satisfacción en cada una de las áreas que se desarrolla el ser humano (Ellickson \& Logsdon, 2002; Moyano \& Ramos, 2007; Boumans, 2012; Belias, Koustelios, Aspridis, Varsanis, \& Koffas, 2016; Marrugo, Landazal, Sierra, \& Fuentes, 2018).

A pesar de que se puede tener mayor satisfacción en algunas áreas de la vida y en otras menos satisfacción, se supone que cada área del ser humano influye sobre las otras; es decir, la satisfacción laboral, puede influir en la satisfacción personal o viceversa; esto, puesto que el ser humano es integral y está constituido de muchos roles, 
actividades y ambientes en los que se desarrolla, es de considerar además que al ser subjetiva puede variar dependiendo del estado de ánimo del individuo (Gamboa, 2010; Belias et al., 2016; Platis \& Zoulias, 2017).

La satisfacción en general tiene que ver con las actitudes que se tengan hacia los diferentes aspectos de su vida. La satisfacción es multifactorial, por lo tanto, los factores que pueden causar mayor satisfacción en un individuo no necesariamente son los mismos que causan satisfacción en otro (Boumans, 2012; Cárdenas, López, \& Fraire, 2013; Carrillo, Solano, Martínez, \& Gómez, 2013; Chatterjee, Clark, Davis, \& Toher, 2017).

La satisfacción laboral ha sido uno de los temas más estudiados, puesto que una persona activa laboralmente, dedica un tercio de la vida al trabajo. Por lo tanto, la satisfacción laboral es uno de los ambientes en los que se desarrolla el ser humano durante más tiempo y además se considera que este eje de la satisfacción influye en la percepción de la satisfacción personal y de aspectos de la vida en general (Benedicto, 2013; Carrillo et al., 2013; Arce, 2015a; Dobrow, Ganzach, \& Liu, 2016).

En Costa Rica existen algunos estudios locales relacionados con el nivel de satisfacción; algunos de ellos están realizados en el personal de una universidad estatal la cual es la población de interés de este estudio. Estos estudios reportan que los niveles de satisfacción personal y con el ámbito nacional, se encuentran en niveles medios, presentando cierta disconformidad en estas áreas (Arce, 2015a). Es importante recalcar que a nivel mundial la escala de satisfacción o felicidad está determinada por otras variables de tipo económico y de índices de salud, por lo cual se dificulta encontrar referencias que expongan de forma similar lo que se presenta en este estudio. Se encontraron además diversas investigaciones del nivel de satisfacción en diversos campos o ramas, que permiten sustentar esta investigación. El objetivo principal del estudio es comparar el nivel de satisfacción de los funcionarios de una universidad estatal costarricense de la Sede Central y los Centros universitarios distribuidos en todo el país.

\section{MATERIALES Y MÉTODOS}

La metodología de este estudio está basada en Arce y Rojas (2019).

Instrumento: El instrumento se aplicó en línea con el software LIMESURVEY, se mantuvo activo desde el 01 de octubre de 2017 al 30 de octubre de 2018. Se envió un enlace a través de la dirección de correo electrónico institucional a 3256 funcionarios que laboran en la institución, lográndose obtener 1310 cuestionarios completos que corresponden a un $41 \%$ de la población total. Para efectos de análisis se decidió clasificarlos geográficamente en Sede Central y Centros universitarios.

Procedimiento: Posterior a la sensibilización de la población se envió un correo de agradecimiento que incluía una invitación personalizada para participar en el estudio, en este correo se indicaba un enlace para acceder el cuestionario y responderlo a través de la web. Durante el periodo en que se mantuvo activo el cuestionario se enviaron cinco recordatorios a los funcionarios que no lo habían contestado con el fin de motivar la participación.

Análisis estadístico: La sección de satisfacción consta de tres ejes o dimensiones: satisfacción personal, laboral y nacional. Para el cálculo de cada subíndice hay un conjunto de ítems medidos en una escala ordinal con cuatro opciones de respuesta: muy satisfecho, satisfecho, insatisfecho y muy insatisfecho. A partir de las respuestas, se obtuvo el valor de los subíndices y del índice de satisfacción personal.

Los datos fueron tabulados inicialmente en un archivo tipo hoja de cálculo y luego se elaboró una base de datos utilizándose el software IBM SPSS Statistics versión 20, mediante el cual se realizó el análisis descriptivo que comprende la elaboración distribuciones de frecuencia, tablas de contingencia y cálculo de valores promedio. Se calcula el estadístico de contraste mediante U Mann Whitney (X2) para verificar diferencias en la distribución de las variables incluidas en el análisis, y el estadístico $\mathrm{H}$ de Kruskal-Wallis para comparar los valores obtenidos para el índice y subíndices con respecto a las características sociodemográficas y laborales de los funcionarios.

Ética, conflicto de intereses y declaración de financiamiento: Las autoras declaran haber cumplido todas las directrices éticas pertinentes descritas por Emanuel, Wendler y Grady (2000), las identidades individuales no se presentan y no pueden ser identificadas, los resultados se presentan de manera agregada para los instrumentos completados, tanto durante el estudio como en el manuscrito; que no hay conflictos de interés de ningún tipo, y que todas las fuentes financieras se detallan plena y claramente en la sección de agradecimientos. Asimismo, están de acuerdo con la versión editada final del documento. El respectivo documento legal firmado se encuentra en los archivos de la revista. 


\section{RESULTADOS}

En relación con las características sociodemográficas y laborales de los funcionarios de la Sede Central hay diferencias en los porcentajes de las variables indagadas (Cuadro 1). Hay mayor número de personas menores de 41 años que laboran en los Centros $(Z=-8,392, p<0,01)$. Con respecto a la escolaridad, la mayoría de los funcionarios de la Sede Central poseen universidad completa $(Z=-10,629, p<0,01)$. La mayoría de los funcionarios de la Sede Central ocupa puestos auxiliares $(Z=6,577, p<0,01)$.

En cuanto al Índice de Satisfacción Personal (ISP) la satisfacción personal, aspectos de la vida y aspectos laborales presentan promedios de satisfacción relativamente altos, superiores a $70 \%$ (Cuadro 2), tanto en la Sede Central como en los Centros Universitarios. Sin embargo, para el subíndice de ambiente nacional se obtiene una medición de promedio general inferior a $60 \%$ en los Centros universitarios con una satisfacción media.

Con respecto al ISP, los funcionarios de la Sede Central presentan mayor satisfacción que los funcionarios de Centros Universitarios (U Mann-Whitney $(Z=-9,088$, $p<0,01)$.

En cuanto al Subíndice de Ambiente Nacional, los funcionarios de la Sede Central presentan una satisfacción alta mayor a $70 \%$, mientras que los Centros presentan una satisfacción media $(56,7 \%$ U Mann-Whitney, Z= $-16,812, p<0,01)$.

\section{DISCUSIÓN}

La satisfacción es la suma de diversos factores de todas las áreas del ser humano, se considera que, al existir influencia de un área sobre la otra, es esperable que los grados de satisfacción en diversas áreas de la vida conserven cierto grado de armonía entre ellas. El subíndice de aspectos de la vida, sigue siendo el más relevante en el grado de satisfacción; las relaciones de familia, pareja y comunidad que conforman este subíndice determinan en cierto grado la satisfacción en las demás áreas, creando un balance en el nivel de satisfacción, se considera además que un grado de satisfacción muy alto o muy bajo en una sola área no es determinante, pues el nivel de satisfacción debe analizarse de acuerdo con las áreas evaluadas y de forma integral (Benedicto, 2013; Caillier, 2013; Pineau, 2013; UP-TNS GALLUP, 2013; Gutiérrez, Sapiem, \& Pinon, 2018; Helliwell, Layard, \& Sachs, 2019). Como puede observarse, aunque en los Centros Universitarios el Subíndice de Satisfacción con el Ámbito Nacional presenta una satisfacción media con respecto

CUADRO 1

Distribución porcentual de las características sociodemográficas y laborales de los funcionarios de la Sede Central y de Centros Universitarios, 2018

\begin{tabular}{lcc}
\multicolumn{1}{c}{ Característica } & Sede & $\begin{array}{c}\text { Centros } \\
\text { universitarios }\end{array}$ \\
Sexo & & \\
Femenino & 57,1 & 60,9 \\
Masculino & 42,9 & 39,1 \\
Grupos de edad & & \\
Menos de 30 años & 9,2 & 20,6 \\
De 31 a 40 años & 36,3 & 40,3 \\
De 41 a 50 años & 29,2 & 17,2 \\
Más de 50 años & 25,3 & 21,9 \\
Estado civil & & \\
Soltero & 31,5 & 36,1 \\
Casado & 46,4 & 40,3 \\
Divorciado, separado, viudo & 14 & 13,7 \\
Unión libre & 8,1 & 9,9 \\
Número de hijos & & \\
Ninguno & 41,4 & 37,8 \\
1 hijo & 22,3 & 21,5 \\
2 hijos & 23,5 & 19,7 \\
3 o más hijos & 12,8 & 21 \\
\hline
\end{tabular}

\begin{tabular}{lcc}
\multicolumn{1}{c}{ Característica } & Sede & $\begin{array}{c}\text { Centros } \\
\text { universitarios }\end{array}$ \\
Escolaridad & & \\
Universidad completa & 80,8 & 49,8 \\
Universidad incompleta & & 35,6 \\
Sin formación universitaria & 19,2 & 14,6 \\
Condición laboral & & \\
Propiedad & 58,5 & 63,5 \\
Plazo fijo & 34,7 & 28,8 \\
Otra & 6,8 & 7,7 \\
Antigüedad laboral & & \\
Menos de 1 año & 5 & 6 \\
De 1 a 5 años & 23,5 & 21,9 \\
De 6 a 10 años & 29,5 & 37,3 \\
De 11 a 15 años & 19,5 & 15,5 \\
Más de 15 años & 22,5 & 19,3 \\
Puesto & & \\
Personal profesional & 17,5 & 29,2 \\
Personal auxiliar & 59,9 & 54,9 \\
Personal apoyo & 22,6 & 15,9 \\
\hline
\end{tabular}


CUADRO 2

Valores promedio de los subíndices según características de los funcionarios de la Sede Central y Centros Universitarios de una universidad estatal de Costa Rica, 2018

\begin{tabular}{lcccc} 
Característica & $\begin{array}{c}\text { Índice satisfacción } \\
\text { personal }\end{array}$ & $\begin{array}{c}\text { Subíndice aspectos } \\
\text { de la vida }\end{array}$ & $\begin{array}{c}\text { Subíndice satisfacción } \\
\text { laboral }\end{array}$ & $\begin{array}{c}\text { Subíndice ambiente } \\
\text { nacional }\end{array}$ \\
Sede & 78,1 & 83,2 & 79,8 & 71,4 \\
Centros Universitarios & 73,1 & 82,7 & 80,0 & 56,7 \\
\hline
\end{tabular}

a los demás y en comparación a la Sede Central, el ISP presenta una satisfacción alta mayor al 70\%, al igual que la Sede Central.

En cuanto al Subíndice de Aspectos de la Vida, tenemos que en ambos casos Sede y Centros, es alto, mostrando una ligera diferencia la Sede de los Centros. Este subíndice engloba las variables subjetivas concernientes a la vida familiar, la vida en pareja y la vida comunal; al igual que lo encontrado por otros autores, se destacan la importancia de estas variables con respecto a las demás e incluso se puede afirmar que éstas son más relevantes para la determinación de la calidad de vida de una persona que las relacionadas con aspectos económicos o laborales. Las relaciones de pareja, de familia y de amistad o camaradería comunal, fortalecen la autoestima y brindan una sensación de seguridad y de pertenencia en los individuos, por lo cual, las personas con niveles altos de satisfacción en esta área son más felices y se encuentran más satisfechos con la vida, a pesar de que en otros aspectos la satisfacción sea media o baja; el subíndice de aspectos de la vida es determinante en el grado de satisfacción y el más importante en el ser humano (Vega \& Neira, 2009; Boumans, 2012; Carrillo et al., 2013; Vega \& Hidalgo, 2013; Arce, 2015b; Matud, 2016; Estado de la Nación, 2018; Sharfika, Siti Nor, Md Saiful, Shahrizal, \& Anwar, 2018; Arce \& Rojas, 2019; Dey, Md. Naf, Miah, \& Maruf, 2019).

El grado de satisfacción laboral en la Sede Central y los Centros Universitarios es alta; se muestra una ligera diferencia entre ambos, siendo mayor la satisfacción en los Centros Universitarios, esto podría explicarse como que la vida cotidiana que rodea las áreas rurales es más tranquila compensando el hecho de que el Índice de Desarrollo Humano es menor con respecto a la Sede Central (Helminen \& Ristimaki, 2007; Universidad de Costa Rica, 2011; Bin Bae \& Kim, 2016; Estado de la Nación, 2018; Arce \& Rojas, 2019), podría ser también que algunos factores que determinan esta área se encuentran más claros en las áreas rurales, por ejemplo: las relaciones con sus iguales y jefaturas, la carga de trabajo, la jornada, el salario, la infraestructura, el equipo, así como la claridad de las funciones (Velandia, Ardón,
\& Jara, 2007; Boumans, 2012; Benedicto, 2013; Carrillo et al., , 2013; Arce, 2015a).

En general, Costa Rica se perfila como un país de economía creciente en turismo ecológico lo cual le ha dado renombre a nivel mundial; el acceso universal a la educación y la salud, la posicionan como uno de los países latinoamericanos con mayor proyección económica. Estos indicadores han contribuido a que la perspectiva de la población se muestre optimista y satisfecho a nivel país, sin embargo, existe una brecha en cuanto a la pobreza y la desigualdad de oportunidades en las zonas rurales, lo cual se refleja en el nivel de satisfacción de los Centros con respecto a la sede; las diferencias de seguridad, empleo, educación, pobreza y salud, siguen siendo aspectos donde existen desigualdades. Las asimetrías económicas y de oportunidades presentes en las diversas zonas del país, producen estancamiento del comercio, desempleo y con esto un nivel menor de satisfacción con respecto a la visión país (Universidad de Costa Rica, 2011; Tello, 2015; Estado de la Nación, 2018; OCDE, 2018).

\section{AGRADECIMIENTOS}

Agradecemos a Julián Monge Nájera de la Vicerrectoría de Investigación UNED, por su apoyo, guía y acertados consejos en el desarrollo de esta investigación. A Ligia Bermúdez Mesén de la Vicerrectoría de Investigación UNED, por su apoyo en esta investigación. A todos los funcionarios de la Sede Central y Centros Universitarios que completaron el instrumento.

\section{REFERENCIAS}

Arce, L. (2015a). Satisfacción personal, laboral y geográfica en funcionarios de una universidad estatal de Costa Rica: efecto del sexo, el estado civil, la edad y la educación. UNED Research Journal, 7 (2), 151-155.

Arce, L. (2015b). Nivel de satisfacción con su vida de los funcionarios. UNED Research Journal, 7 (2), 131-135. 
Arce, L., \& Rojas, K. (2019). Satisfacción en Centros universitarios de una universidad estatal de Costa Rica. UNED Research Journal, 11(3), 320-326.

Belias, D., Koustelios, A., Aspridis, G., Varsanis, C., \& Koffas, S. (2016). Personal traits and Job satisfaction of people who work into the Greek banking sector. International Journal of Strategic Innovative Marketing, 3(3), 41-55.

Benedicto, E. T. (2013). Satisfacción laboral y su relación con variables sociodemográficas y laborales y con parámetros de riesgo cardiovscular en trabajadores de Baleares. Medicina Balear, 28(2), 29-34.

Bin Bae, K., \& Kim, D. (2016). The Impact ofDecoupling ofTelework on Job Satisfaction in U.S. Federal Agencies: Does Gender Matter? The American Review of public adminsitration, 46(3), 356-371. DOI: 10.1177/0275074016637183

Boumans, N. (2012). Age-Differences in Work Motivation and Job Satisfaction. The Influence of Age on the Relationships between Work Characteristics and Workers' Outcomes. The international journal of aging and human development, 73(4), 331-350. DOI: 10.2190/AG.73.4.d

Caillier, G. (2013). Satisfaction With Work-Life Benefits and Organizational Commitment/Job Involvement: Is There a Connection? Review of public personnel administration, 33(4), 340-364. DOI: 10.1177/0734371X12443266

Cárdenas, M. C., López, A. M., \& Fraire, A. A. (2013). Relación de los factores en la satisfacción laboral de los trabajadores de una pequeña empresa de la industria metal mecánica. Revista Internacional de Administración y Finanzas, 6(3), 116-128.

Carrillo, G., Solano, R., Martínez, R., \& Gómez, G. (2013). Influencia del género y edad: satisfacción laboral de profesionales sanitarios. Revista Latino-Americana de Enfermagem, 21(6),1314-1320. DOI: 10.1590/0104-1169.3224.2369

Chatterjee, K., Clark, B., Davis, A., \& Toher, D. (2017). The Commuting and Wellbeing Study: Understanding the Impact of Commuting on People's Lives. Bristo, UK: University of the West of England.

Dey, T., Md. Naf, S., Miah, M., \& Maruf, M. (2019). Analyzing the Extents of the Factors Influential to the Level of Job Satisfaction: A Study on Bankers from Dhaka City's Private Commercial Banks. International Journal of Science and Business, 3(1), 72-83.

Dobrow, R. S., Ganzach, Y., \& Liu, Y. (2016). Time and job satisfaction: a longitudinal study of the differential roles of age and tenure. Journal of management, 44(7), 2558-2579. DOI: $10.1177 / 0149206315624962$

Duarte, S., Aguirre, J., \& Jarquín, G. (2014). Factores que influyen en la satisfacción laboral de los empleados de ADARA S.A. Revista electrónica de investigación en ciencias económicas, 2(4), 1-14. DOI: 10.5377/reice.v2i4.1723

Ellickson, M., \& Logsdon, K. (2002). Determinants of Job Satisfaction of Municipal Government Employees.
Public Personnel Management, 33(3), 343-358. DOI: 10.1177/009102600203100307

Emanuel, E., Wendler.D, \& Grady, C. (2000). What makes clinical research ethical? Revista JAMA, 283(20), 2701-2711. DOI: 10.1001/jama.283.20.2701

Estado de la Nación. (2018). Décimocuarto Informe Estado de la Nación en Desarrollo Sostenible, Bienestar Subjetivo. San José, Costa Rica: Merck Company Foundation.

Gamboa, E. (2010). Satisfacción laboral: Descripción teórica de sus determinantes. Revista psicológica científica, 12, 12-16.

Gutiérrez, M., Sapiem, A., \& Pinon, L. (2018). Telework as a Labor Satisfaction Strategy. Findingsin in Emerging Economies. European Scientific Journal, 14(22), 1-12. DOI: 10.19044/esj.2018.v14n22p1

Helliwell, J., Layard, R., \& Sachs, J. (2019). World of happiness report. Nueva York, EEUU: United Nation.

Helminen, V., \& Ristimaki, M. (2007). Relationships between commuting distance, frequency and telework in Finland. Journal of Transport Geography, 15(5), 331-342. DOI: 10.1016/j.jtrangeo.2006.12.004

Marrugo, E. A., Landazal, M. S., Sierra, C. A., \& Fuentes, E. A. (2018). Factores asociados a la satisfacción laboral en docentes de una institución de educación superior del Caribe Colombiano . Revista Espacios, 39(2), 1-14.

Matud, A. (2016). Evaluación de la satisfacción con el rol laboral en mujeres y hombres. Salud de los trabajadores, 24(1), 17-26.

Moyano, D., \& Ramos, A. (2007). Bienestar Subjetivo: midiendo satisfacción vital, felicidad y salud en población. Revista Universum, 22(2),177-193. DOI: 10.4067/ S0718-23762007000200012

OCDE. (2018). Estudios económicos de la OCDE, Costa Rica 2018. París, Francia: OECD. DOI: 10.1787/ eco_surveys-cri-2018-graph76-en

Pineau, L. (2013). The influence of personal and workplace resources on new graduate nurses'job satisfaction. Journal of nursing, 23(2), 190-199. DOI: 10.1111/jonm.12113

Platis, C., \& Zoulias, E. (2017). Organization Style and Its Effect on Employee Satisfaction and Personal Performance. In A. Kavoura, D. Sakas, \& P. Tomaras (eds), Strategic Innovative Marketing (pp. 151-158). Nueva York, EE.UU: Springer, Cham. DOI:10.1007/978-3-319-56288-9_2

Sharfika, B. R., Siti Nor, B. A., Md Saiful, A., Shahrizal, B., \& Anwar, R. (2018). Influence ofEmployee Empowerment,Teamwork, and Incentive on Employee's Job Satisfaction. American Journal of Social Sciences and humanities,1(2), 40-47. DOI: 10.20448/801.31.40.47

Téllez, J. A. (2011). Organización del trabajo y satisfacción laboral; un estudio de caso en la industria del calzado. Nova Scientia, 4(7), 177-204. 
Tello, C. (2015). Satisfacción residencial: un concepto de calidad de vida en la ciudad Montreal. Revista de Ciencias Sociales, $148,67-81$.

Universidad de Costa Rica. (2011). Atlas de Desarrollo Humano 2011. San José, Costa Rica: PNUD-UCR.

UP-TNS GALLUP (Universidad de Palermo-Trasnsparent Network Substrate). (2013). Estudio sobre la felicidad. Informe institucional. Universidad de Palerm, Buenos Aires, Argentina.
Vega, M. M., \& Hidalgo, J. F. (2013). Estudio de la relación entre la satisfacción laboral y desempeño de los trabajadores de las ferias libres. Contaduría y administración, 58(2),40-60.

Vega, M. M., \& Neira, N. J. (2009). Análisis de la satisfacción y el desempeño laboral en los funcionarios de la Municipalidad de Talcahuano. Ciencia y trabajo, 54, 159-165.

Velandia, F., Ardón, N., \& Jara, M. (2007). Satisfacción y calidad, análisis o no de la equivalencia de los términos. Revista Gerenciaol y Políticas de Salud, 6(13), 139-168. 